\title{
Mineraçãào
}

\section{Análise da variabilidade de teores e sua incorporação no planejamento de lavra}

\author{
Grade variability analysis and \\ its use in mine planning
}

\author{
Rodrigo de Lemos Peroni \\ Eng. Minas, MSc., \\ Dr. Eng., Prof. Adjunto do \\ Departamento de Eng. de Minas UFRGS. \\ peroni@ufrgs.br
}

\section{João Felipe C. L. Costa}

Eng. Minas, MSc.,

PhD., Prof. Associado do

Departamento de Eng. de Minas UFRGS.

jfelipe@ufrgs.br

\section{Jair Carlos Koppe}

Eng. Minas, MSc.,

Dr. Eng., Prof. Titular do

Departamento de Eng. de Minas UFRGS.

jkoppe@ufrgs.br

\section{Resumo}

Esse estudo apresenta os resultados obtidos usando técnicas de simulação geoestatística combinadas com técnicas de otimização e sequenciamento para análise do impacto de incerteza dos teores no planejamento de lavra. Abordagens tradicionais utilizam modelos estimados por técnicas geoestatísticas (e.g. Krigagem Ordinária, Krigagem dos Indicadores) ou mesmo técnicas clássicas de estimativa para avaliação de depósitos minerais (e.g. Inverso do Quadrado da Distância, Polígonos, Média Móvel). Da mesma maneira, ao se avaliarem projetos de mineração, normalmente, são feitas análises de sensibilidade aos parâmetros econômicos e é desconsiderada a incerteza associada ao modelo geológico. Esse estudo demonstra que os teores podem ter impacto na incerteza de projetos, tanto no aspecto de projeto de cava, quanto no aspecto de viabilidade econômica (análise de sensibilidade).

Palavras-chave: Planejamento, simulação, incerteza geológica, avaliação de reservas, sensibilidade.

\begin{abstract}
This study presents the results obtained using geostatistical simulation techniques combined with pit optimization and sequencing to assess the grade uncertainty impact in mine planning and reserve assessment. Traditional approaches use an estimated model by geostatistical methods (Ordinary Kriging, Indicator Kriging) or even classical methods of estimation (IPD, polygons, moving average). In the same manner, to evaluate mining projects, analyses considering economical sensitivity aspects were made, disregarding the uncertainty of the geological model. This study demonstrates that grade estimation can have a relevant impact on the uncertainty over mining projects, even in the open pit design, i.e. in the feasibility study.
\end{abstract}

Keywords: Planning, simulation, geological uncertainty, reserves assessment, sensitivity.

\section{Introdução}

Esse estudo apresenta os resultados obtidos usando técnicas de simulação geoestatística combinadas com técnicas de otimização e sequenciamento para análise do impacto de incerteza dos te- ores no planejamento de lavra. Tradicionalmente, são utilizadas abordagens utilizando um único modelo médio estimado por técnicas geoestatísticas (e.g. Krigagem Ordinária - Matheron, 1975, 
Krigagem dos Indicadores) ou mesmo técnicas clássicas de estimativa para avaliação de depósitos minerais (Inverso do Quadrado da Distância, Polígonos, Média Móvel). Da mesma maneira, ao se avaliarem projetos de mineração, normalmente, são feitas análises de sensibilidade aos parâmetros econômicos, desconsiderando a incerteza associada ao modelo geológico.

\section{Aplicação da metodologia}

Foi selecionado um depósito de ouro para se aplicar a metodologia. O banco de dados contempla 94 furos de sondagem cobrindo uma área superfi-
A proposta desse trabalho - e sua principal contribuição -, exatamente, demonstrar que os teores podem ter impacto na incerteza de projetos, tanto no aspecto de projeto de cava, quanto no aspecto de viabilidade econômica. Para a determinação de um cenário de referência, foi construído um modelo geológico e de teores a partir de informações geoposicionadas de um depósito de ouro.

cial de, aproximadamente, $240.000 \mathrm{~m}^{2}$. Os dados de sondagem estão espaçados em uma malha quasi-regular de aproximadamente $40 \times 40 \mathrm{~m}$. A área alvo da
Para construir cenários de incerteza, foram utilizados algoritmos de simulação estocástica e avaliadas as oscilações sobre o modelo estimado para o depósito tido como referência. Como resultado, foi avaliada a significância do impacto da incerteza geológica frente a outras variações de parâmetros na quantificação de reservas minerais e viabilidade econômica de projeto.

\section{Modelo geológico e características dos dados}

Foram identificados, inicialmente, sete sub-domínios geológicos dentro do banco de dados, sendo que destes sete, três são domínios estéreis e quatro são domínios mineralizados. Dos domínios mineralizados, foi selecionado apenas um para a estimativa e a simulação. Tal decisão foi tomada levando em consideração a representatividade e a quantidade de informações então obtidas. O objetivo foi o de produzir análises de continuidade espacial adequadas, levando em consideração uma quantidade mínima de dados. O resumo estatístico dos dados

\begin{tabular}{c|c}
\hline \multicolumn{2}{c}{$\mathrm{Au}<40 \mathrm{~g} / \mathrm{t}$} \\
\hline Média & 2,85 \\
\hline Mediana & 2,06 \\
\hline Moda & 1,16 \\
\hline Variância da amostra & 14,25 \\
\hline Curtosidade & 44,84 \\
\hline Espalhamento & 6,02 \\
\hline Valor mínimo & 0,11 \\
\hline Valor máximo & 36,11 \\
\hline No. de dados & 297 \\
\hline
\end{tabular}

pesquisa está concentrada no centro do levantamento topográfico, se estendendo pela região norte, atravessando um vale e subindo na encosta sul/sudoeste da área.

amostrais, para o domínio mineralizado identificado, dentro do depósito, está apresentado na Tabela 1. As amostras estão regularizadas verticalmente, respeitando os domínios geológicos que foram identificados anteriormente a intervalos de $2 \mathrm{~m}$.

Tabela 1

Sumário estatístico dos dados do domínio de interesse para a variável Au.

\section{Estimativa preliminar de recursos geológicos}

Foi realizada uma estimativa preliminar para se avaliar a quantidade total de minério no depósito.

A Tabela 2 apresenta os resultados individualizados por domínio geológi- co, expressos em termos de volume e de massa, considerando que as diferentes litologias do depósito possuem densidades médias iguais.

Considerando a proporção do do- mínio LITO=7, dentro do contexto do trabalho e do depósito, selecionou-se, apenas, esse domínio, para se desenvolver e se aplicar a metodologia.

\begin{tabular}{c|c|c|c}
\hline LITO & Volume $\left(\mathbf{m}^{3}\right)$ & Densidade $\left(\mathbf{t} / \mathbf{m}^{3}\right)$ & Massa $(\mathbf{t})$ \\
\hline 7 & 958.012 & 2,5 & 2.395 .012 \\
\hline 11 & 364.024 & 2,5 & 910.060 \\
\hline 9 & 70.169 & 2,5 & 175.422 \\
\hline 8 & 21.306 & 2,5 & 53.265 \\
\hline Total & 1.413 .511 & 2,5 & 3.533 .776 \\
\hline
\end{tabular}

\section{Variografia para o domínio de interesse}

A Tabela 3 apresenta o modelo variográfico utilizado para introdução dos parâmetros de continuidade espacial, para a estimativa de teores, e fornece,

\begin{tabular}{c|c|c|c|c|c|c|c}
\hline Domínio & C0 & Patamar 1 & Ângulo & Mergulho & Alcance Máx. & Patamar 2 & Alcance Mín. \\
\hline LITO=7 & 0.35 & 0.65 & 45 & -20 & 220 & 1 & 176 \\
\hline
\end{tabular}

também, orientação para a estratégia de busca a ser utilizada.

Tabela 3 - Resumo dos parâmetros de ajuste variográfico. 


\section{Construção da Função-Benefício}

Para se avaliar, as cavas finais obtidas, a partir de cada realização, foi utili-

Para a análise de cenários, foram

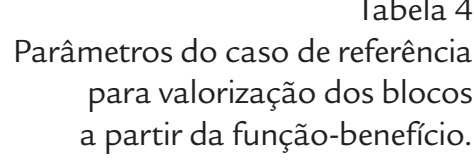

O cálculo do teor de corte foi exe-
Onde:

Custos Op. - custos operacionais

Rec - Recuperação global do processo de beneficiamento. zada a mesma função benefício usada sobre o modelo estimado usando krigagem

Função-Benefício $=$ Receitas - Custos

assumidos custos e parâmetros econômi-

cos expressos conforme a Tabela 4:

\begin{tabular}{c|c|c}
\hline & Sigla & Valor \\
\hline Custo de Lavra & $\mathrm{C}_{L}$ & $2,00 \mathrm{US} \$ / \mathrm{t}$ \\
\hline Custo de Beneficiamento & $\mathrm{C}_{\mathrm{B}}$ & $5,00 \mathrm{US} \$ / \mathrm{t}$ \\
\hline Custos Operacionais & Custos Op. $=\mathrm{C}_{\perp}+\mathrm{C}_{\mathrm{B}}$ & $7,00 \mathrm{US} \$ / \mathrm{t}$ \\
\hline Preço de Venda & $\mathrm{S}$ & $600 \mathrm{US} \$ / \mathrm{oz}$ \\
\hline Preço de Venda & $\mathrm{S}$ & $19,29 \mathrm{US} \$ / \mathrm{g}$ \\
\hline Recuperação global & $\mathrm{Rec}$ & $80 \%$ \\
\hline
\end{tabular}

cutado conforme a Equação 2, que repre-

senta o teor de corte no equilíbrio.

$$
\text { Teor de corte }=\frac{\text { Custos Op. }}{\operatorname{Rec} * S}
$$

$S$ - Preço de venda do concentrado.

O resultado de teor de corte, para o caso de referência, está apresentado na Equação 3 e se configura através do valor ordinária, conforme a Equação 1:

$B C O G=\frac{C_{L}+C_{B}}{\operatorname{Rec}^{*} S}=\frac{2+5}{0.8 * 19,29}=0,454 \mathrm{~g} / t$

de decisão, se o bloco for considerado estéril ou minério, dentro do planejamento de lavra.
A sequência operacional utiliza a rotina de simulação sequencial Gaussiana - SGsim (Isaaks, 1990, Deutsch \& Journel, 1998) adaptada para a geração de modelos em domínios irregulares (Peroni, 2002). A modificação depende da existência de um arquivo de comparação contendo a informação dos blocos que deverão ser simulados e dos que deverão ser ignorados no processo (identificador "0" para desconsiderar e " 1 " para considerar). Conforme a rotina, o resultado é um vetor de dados contendo todas as realizações geradas. Segue-se o processo com o pós-processamento, para geração do cenário da média de referência, representado pelo E-type (esperança matemática das realizações simuladas), e, posteriormente, faz-se a adição de coordenadas, para importação, e faz-se, ainda, análise em programa específico de mineração.

Foram geradas 50 realizações para o depósito. Para se examinarem as realizações, elas foram classificadas segundo uma rotina também adaptada por Peroni (2002), chamada RANKTRIM. Os resultados são exportados na forma de um arquivo-texto, contendo os parâmetros média e variância de cada um dos cenários de simulação. Tais cenários fo- ram classificados em ordem ascendente da menor para a maior média.

A análise desse resultado permite a seleção de cenários para avaliação da incerteza. Cada uma das realizações selecionadas caracteriza um provável cenário do depósito, usando-se a simulação geoestatística. Isso significa que cada modelo é uma representação possível do depósito por ser um retrato matemático/estatístico, reproduzindo os parâmetros média e variância dos dados originais e, também, como sua continuidade espacial expressa pelo variograma.

O pós-processamento permite que sejam avaliados os cenários de forma conjunta e global, sendo que um dos resultados é de particular interesse. $\mathrm{O}$ chamado E-type da simulação corresponde à esperança matemática dos teores de cada bloco do depósito em questão, construído a partir da média aritmética de todos cenários simulados para cada um dos blocos. Esse modelo se assemelha muito com modelos estimados, usando-se técnicas de krigagem ordinária. Dessa forma, o referido modelo foi considerado como modelo de referência, para as análises de variação de teores e de sensibilidade.

Os arquivos de resultados (E-type e cenários simulados individualmente) adicionados de coordenadas são valorizados conforme a função-benefício. Após a valorização dos blocos, cada um dos cenários selecionados foi integrado a um programa de otimização de cava e de sequenciamento de lavra, para se avaliar o resultado econômico que cada cenário produz frente ao processo.

Resgatando as médias das simulações apresentadas na Tabela 1, observa-se que a variação de teores médios simulados apresentaram alguns resultados extremos, chegando-se até $+/-15 \%$ de variação em torno do cenário médio (E-type). Considerando que $96 \%$ das realizações ficaram concentradas em um intervalo de variação de $+/-10 \%$, tal intervalo foi adotado como os limites extremos. A variação de teores foi, então, comparada com variações do preço de venda, recuperação metalúrgica e custos operacionais para determinar a relevância do aspecto incerteza de teores.

As variáveis de comparação foram analisadas segundo incrementos e decrementos de até $+/-30 \%$ a intervalos regulares de $5 \%$, em relação ao caso de referência (E-type). Para cada uma das análises, foi variado um dos parâmetros e os demais foram mantidos constantes.

Para o preço de venda, foi adotado, 
como preço de longo prazo do caso de referência, o valor de US\$600/oz. Sobre o valor determinado, foram executados os incrementos e decrementos até o limite de $+/-30 \%$. Esse valor, normalmente, é definido pelas empresas a partir de análises históricas do valor de venda do bem mineral, bem como das tendências de mercado para projeção de valores futuros. A despeito de o preço atual (2011) do ouro ser muito superior ao valor praticado nes-

\section{Otimização de cava}

Normalmente é visada a maior lucratividade possível dentro do contexto de maximização do valor presente líquido (VPL) do projeto. Segundo essa concepção de otimização, existe uma série de algoritmos desenvolvidos que se propõem a atingir os objetivos anteriormente apresentados, porém os métodos que alcançaram a maior popularidade e consequente implementação computacional foram a técnica dos cones flutuantes (Pana \& Carlson, 1966; David et al., 1974); (Lemieux, 1979) e o algoritmo de Lerchs-Grossmann (Lerchs \& Grossmann, 1965). Desde sua introdução, em 1965, o algoritmo de Lerchs-Grossmann (LG) tem sido reconhecido como o único algoritmo que fornece a solução ótima para o problema de projeto de cava a céu aberto. Vários pesquisadores têm desenvolvido algoritmos e métodos alternativos para solucionar, principalmente, o problema de desempenho computacional do algoritmo original de LG, particularmente para problemas de grande número de blocos. Entre esses, autores como

\section{Planejamento de lavra}

Em planejamento de lavra convencional, após ter sido criado um modelo de teores, este é avaliado economicamente e é definido o formato da cava final. O próximo estágio é determinar como a mina será desenvolvida e como produzir uma sequência de extração adequada. Dentro do sequenciamento de lavra, determina-se a vida útil da mina que é dada pela razão de produção da mina, pelo projeto dos avanços operacionais e pela determinação

\section{Análise dos resultados}

Os resultados obtidos, nesse projeto, estão compilados ao longo dessa seção. Inicia-se apresentando os resultados se estudo, o preço utilizado não invalida o estudo, pois as análises foram efetuadas da mesma forma como foram feitas para os cenários de sensibilidade sobre esse mesmo preço de venda.

O segundo parâmetro de sensibilidade analisado foi o custo operacional. Considerando que o custo operacional tem impacto, tanto nos blocos de minério, quanto nos blocos estéreis, foram individualizados os custos totais que im-

Zhao e Kim (1992) introduziram um novo algoritmo de projeto baseado também em teoria gráfica, reduzindo, significativamente, o número de arcos comparado com o algoritmo de LG. Huttagosol e Cameron (1992) apresentaram uma formulação para o problema de modelo de transporte, comparando os resultados obtidos com as abordagens de máximo fluxo (Ford \& Fulkerson, 1956) e os métodos gráfico de LG e cones flutuantes.

A geração de um projeto de cava final tem sido o primeiro passo de um planejamento de produção. Como resultado, muitos algoritmos de projeto de cava têm sido desenvolvidos, tanto na categoria dos métodos heurísticos, método dos cones flutuantes (Lemieux, 1979), na categoria dos métodos matemáticos, tal como o método gráfico de Lerchs-Grossmann (Lerchs \& Grossmann, 1965; Zhao \& Kim, 1991) e o algoritmo do fluxo de rede.

Invariavelmente, esses algoritmos contam com modelos de blocos e, para cada bloco, é atribuído um valor mo-

da sequência de extração dos blocos. Também, identificam-se guias gerais, projetando-se as atividades de planejamento de curto e longo prazo. O papel do engenheiro de minas é de interpretar as opções menos atrativas, eliminandoas. Deve, então, focar nas alternativas com maior viabilidade.

Existem dois tipos de planejamento de produção segundo Couzens (1979):

1. Operacional ou planejamento de curto prazo: necessário para o funciona- pactam sobre os blocos de minério e os custos de lavra que impactam somente sobre os blocos estéreis.

O terceiro parâmetro de sensibilidade avaliado foi a recuperação metalúrgica. Considerando que, para o caso de referência, foi utilizada uma recuperação de $80 \%$ do Au contido e a variação foi de $+/-25 \%$, limitou-se a variação, de outra maneira seriam geradas recuperações maiores do que $100 \%$.

netário líquido (de ganho ou de perda). Desde que o valor do bloco seja uma função do preço do minério e dos custos de processo, a cava projetada com esse conjunto de valores fixos, torna-se obsoleta com o passar do tempo, quando os preços ou os custos sofrerem mudanças. Para acomodar as mudanças nos valores dos blocos, a "análise dos limites da cava" tem sido praticada. Nessa análise, os parâmetros econômicos são, sistematicamente, modificados, um de cada vez, e uma cava é projetada após cada mudança. A saída da análise de projeto é uma série de cavas na qual cada cava possui seu próprio potencial de ser minerada sob condições econômicas específicas. De fato, a análise de projetos de cava nada mais é do que uma parametrização respeitando os parâmetros econômicos (Wang \& Sevim, 1995). Nesse estudo, foi utilizado um programa que faz uso do algoritmo de Lerchs-Grossmann para criação da cava final (ao selecionar a maximização do VPL).

mento de uma mina em operação.

2. Planejamento de produção de longo prazo: usualmente realizado para estudos de viabilidade e financiamento. Isto subentende trabalhos de avaliação de reservas e definição de cava final, elementos determinantes no processo de tomada de decisão.

Nesse estudo, os resultados foram analisados em termos do segundo item descrito. gerados pelo chamado caso de referência, que corresponde ao modelo E-type com preço de venda de US\$600/oz, com cus- to operacional total de US\$7,00/t (US\$ $2,00 /$ t para lavra e US $\$ 5,00 /$ t para o beneficiamento) e recuperação metalúr- 
gica de $80 \%$. Esse resultado representa o modelo de trabalho (E-type, que é usado pela maior parte das empresas do setor mineral, para avaliar os resultados financeiros de um determinado projeto.

Tabela 5

Resultados com base na cava final, usando-se o modelo E-type da simulação.

O sequenciamento obtido pela cava final é calculado com base nas fases criadas para se o resultado, ao passo que o VPL obtido pelo sequenciamento é o VPL que respeita os avanços operacionais e é sequenciado conforme a razão de produção estabelecida.

Segundo Taylor (1977), é possível

onde:

Rec - Corresponde aos recursos globais expressos em milhões de toneladas.

Isto implica uma razão de produção aproximada de 246.000 a 370.000 t/ano. Adotou-se uma cota de 250.000 t/

Figura 1

Representação espacial da cava final para o modelo E-type da simulação.

\section{Análise dos cenários simulados}

Os cenários simulados apresentados a seguir estão sempre comparados ao caso de referência (E-type). A sequência de gráficos que é apresentada demonstra o impacto do cenário simulado face ao cenário médio. Considerando que o modelo E-type da simulação produziu uma média de $2,87 \mathrm{~g} / \mathrm{t}$, esse resultado foi comparado com as demais médias para selecionar as realizações que mais se aproximassem do valor do incremento/ decremento de $+/-5 \%$ das análises de sensibilidade para os dados de preço de ven-
É importante lembrar que existem mais dois aspectos que foram definidos como constantes para todos os cenários e que têm impacto direto nos resultados econômicos. O ângulo global de talude, que foi

\begin{tabular}{c|c}
\hline ETYPE & Resultados \\
\hline VPL $($ US\$) & 54.836 .062 \\
\hline Total Ore $(\mathrm{t})$ & 5.177 .782 \\
\hline Total Waste $(\mathrm{t})$ & 25.944 .420 \\
\hline Strip Ratio $(\mathrm{t} / \mathrm{t})$ & 5,01 \\
\hline $\mathrm{AU}(\mathrm{g} / \mathrm{t})$ & 2,87 \\
\hline
\end{tabular}

calcular uma taxa ótima de extração de um corpo mineral. Para isto, o conhecimento da massa total de recursos e o conhecimento de seus respectivos teores, além de todos os custos e preços de produtos ao longo da duração do projeto, são requeridos. A razão de produção estabelecida foi calculada,

Vida útil $_{\text {Taylor }}=(1 \pm 0,2) * 6,5 * \sqrt[4]{\operatorname{Rec}}$

ano, para valorizar o VPL do projeto, e, também, para se fazer o sequenciamento de lavra.

A Figura 1 apresenta uma ilustração da cava final, que é o resultado da otimização, usando-se o modelo E-type definido como 50, e a taxa de desconto anual, que foi definida em $8 \%$. Os resultados do sequenciamento sobre o cenário médio (E-type) está apresentado na Tabela 5 . da simulação. As cores apresentadas na imagem possuem relação apenas com a elevação, e foram apresentadas apenas para facilitar o efeito de visualização e para se ter uma noção de profundidade, não configurando teores.

usando-se a regra de Taylor, conforme Equação 4, tomando como base os recursos globais determinados para o domínio $\mathrm{LITO}=7$, conforme a Tabela aplicando-se o teor de corte. Como resultado, obteve-se um projeto entre 6,5 a 10 anos de vida útil.

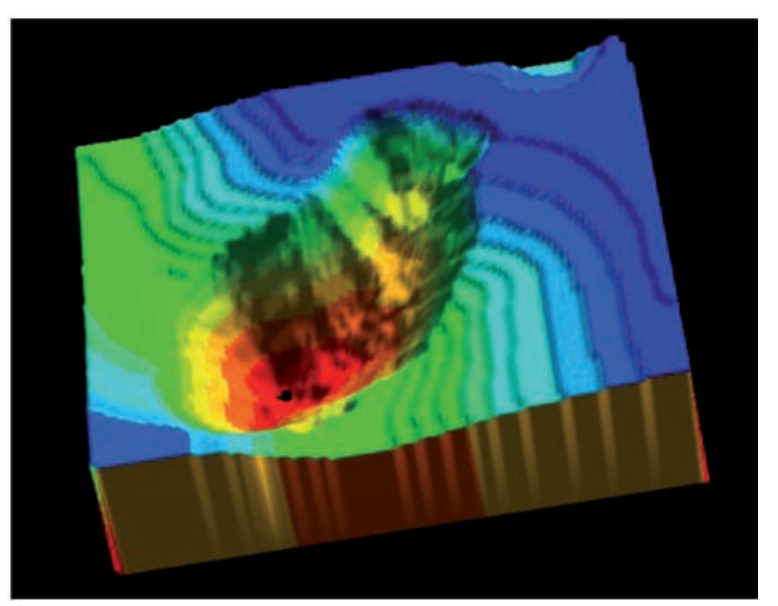

da, custo e recuperação. Sendo assim, a Tabela 6 apresenta, na coluna "No. Realização", o modelo simulado que mais se aproxima do valor de desvio percentual, apresentando o que seria o valor exato na coluna "Média Calculada" e o valor real obtido para a realização do cenário simulado na coluna "Média da Realização".

As realizações geradas demonstram que a possibilidade de se obter um cenário com variações de teores além dos limites de $+/-5 \%$ é de mais de $30 \%$, o que significa que em 16 das 50 realizações geradas, obtiveram-se valores acima ou abaixo dos limites apresentados na Tabela 6.

As Figuras 2 e 3 demonstram os resultados obtidos entre diferentes cenários simulados em comparação com o modelo E-type. Observa-se, nos gráficos, que existem variações entre os cenários simulados e o caso de referência (E-type). Por exemplo, na Figura 2, temos os valores de teores médios e o respectivo VPL do projeto, considerando que os teores fossem assumidos como os teores simulados para cada um dos oito cenários apresen- 


\begin{tabular}{|c|c|c|c|c|}
\hline & Variação & $\begin{array}{c}\text { Média } \\
\text { Calculada }\end{array}$ & No. Realização & $\begin{array}{c}\text { Média da } \\
\text { Realização }\end{array}$ \\
\hline & $20 \%$ & 3,44 & Além dos limites obtidos & - \\
\hline & $15 \%$ & 3,30 & REAL48 & 3,30 \\
\hline & $10 \%$ & 3,15 & REAL24 & 3,18 \\
\hline & $5 \%$ & 3,01 & REAL45 & 3,04 \\
\hline \multicolumn{5}{|c|}{ E-type teor médio 2,87} \\
\hline & $-5 \%$ & 2,72 & REAL15 & 2,73 \\
\hline & $-10 \%$ & 2,58 & REAL22 & 2,63 \\
\hline & $-15 \%$ & 2,44 & REAL17 & 2,46 \\
\hline & $-20 \%$ & 2,30 & Além dos limites obtidos & - \\
\hline
\end{tabular}

tados. Para o caso das Figuras 3A e 3B, estão apresentadas, respectivamente, a massa de minério e a relação estéril minério (REM), para os mesmos cenários.

Essas figuras resumem o resultado diferenciado e o impacto que pode ocorrer mediante a seleção de um cenário médio estimado e mediante as possibilidades de
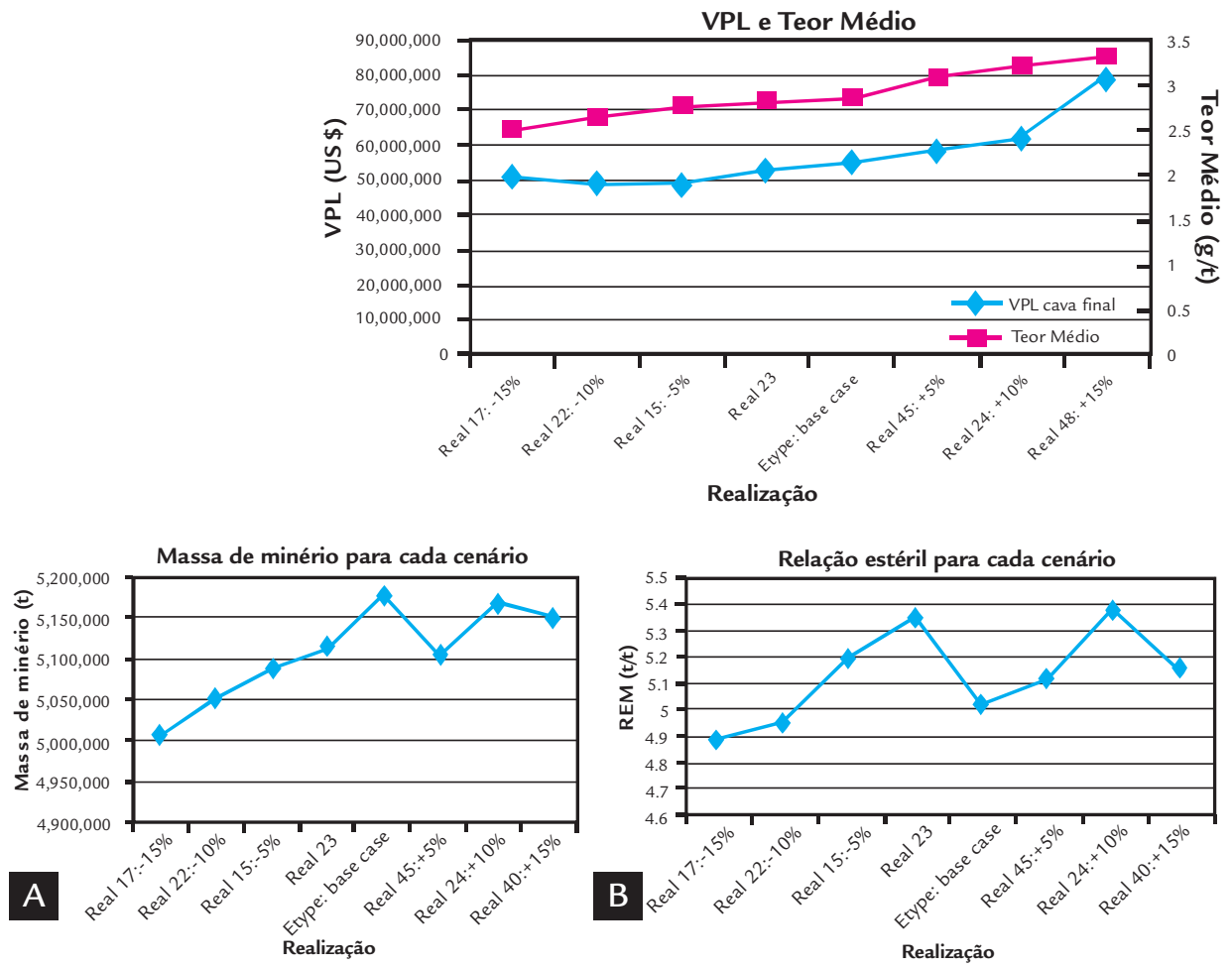

\section{Análise de sensibilidade}

Para cada um dos resultados, foi aplicada a metodologia de variação dos parâmetros, sendo feitas uma posterior exportação e uma análise, dentro programa de otimização e sequen- ciamento. Para finalizar, as análises de sensibilidade foram construídos mais 34 cenários, além dos 8 cenários selecionados entre as realizações geradas pelo algoritmo de simulação. Estão su-

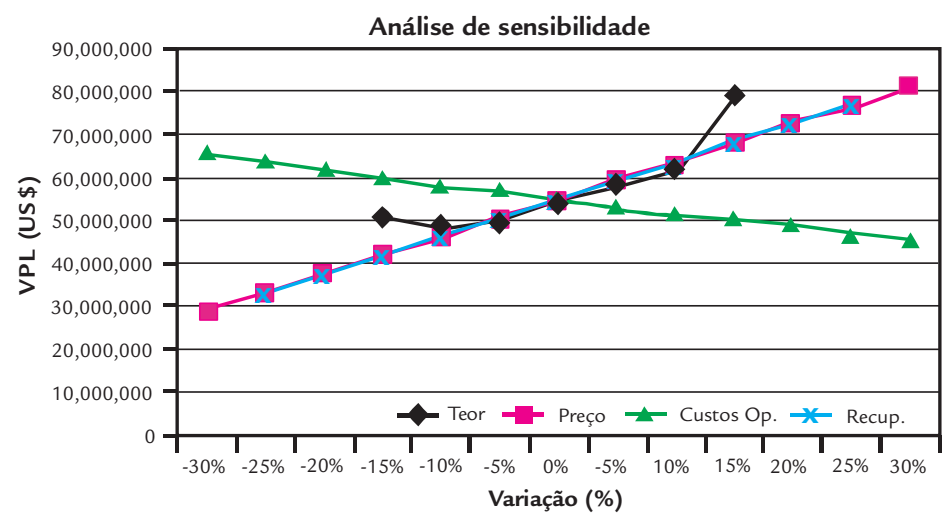

Tabela 6

Cenários simulados selecionados para avaliação de incerteza.

oscilação de teores e dos resultados em tonelagem, quando consideradas possíveis representações do depósito e a variabilidade associada à incerteza geológica.

Figura 2

Teor médio e respectivo VPL para cada um dos cenários simulados e E-type.

Figura 3

Tonelagem total de minério para cada um dos cenários e E-type (A) e REM global para cada cenário simulado e E-type (B).

marizados, na Figura 4, os resultados referentes à análise de sensibilidade ao preço de venda aos custos operacionais e a sensibilidade à variações na recuperação metalúrgica.

Figura 4

Análise de sensibilidade aos parâmetros econômicos comparados. 
A Tabela 7 apresenta o sumário dos resultados obtidos nas comparações realizadas entre o caso de referência e os cenários de sensibilidade testados, demonstrando as variações absolutas e os percentuais obtidos para cada um dos

Tabela 7

Análise de sensibilidade do impacto da variação dos parâmetros sobre o VPL.

Figura 5

Análise de sensibilidade aos parâmetros econômicos.

\section{Comentários finais}

A particularidade do caso de estudo selecionado refere-se, justamente, ao fato de se tratar de um depósito de altos teores. Dessa maneira, as variações dos parâmetros selecionados tiveram pequeno impacto sobre o teor de corte, considerando a abordagem do teor de corte no equilíbrio. Esse fato faz com que tenhamos mantido aproximadamente constante a quantidade de minério existente no depósito, bem como a relação estéril minério e o teor médio do depósito para cada um dos cenários.

Apesar de as variações de incerteza sobre os teores terem ficado em uma faixa restrita de $+/-15 \%$, com base na média das simulações, e ain$\mathrm{da}$ se tais teores forem restringidos ao intervalo de $96 \%$ das ocorrências a apenas $+/-10 \%$, verifica-se que a casos estudados.

A Figura 5 apresenta resultados similares aos resultados da figura anterior, porém acrescenta a informação da variação representada pela linha vermelha. Pode-se observar que a linha apresenta declividade diferente das demais linhas dos outros cenários, o que significa que ordens de variação sobre o parâmetro vão refletir, diferentemente, sobre os resultados econômicos do projeto (VPL).

\begin{tabular}{c|c|c|c|c|c|c|c|c|c}
\hline \multicolumn{10}{c}{ VPL perc. } \\
\hline & Variação & Teor & Preço & Custos Op. & Recup. & Teor & Preço & Custos Op. & Recup. \\
\hline 1 & $-30 \%$ & - & $28,829,786$ & $65,802,494$ & - & - & $-47.43 \%$ & $20.00 \%$ & - \\
\hline 2 & $-25 \%$ & - & $32,979,025$ & $63,739,169$ & $32,976,718$ & - & $-39.86 \%$ & $16.24 \%$ & $-39.86 \%$ \\
\hline 3 & $-20 \%$ & - & $37,598,217$ & $61,956,905$ & $37,598,217$ & - & $-31.44 \%$ & $12.99 \%$ & $-31.44 \%$ \\
\hline 4 & $-15 \%$ & $50,719,155$ & $42,243,247$ & $59,955,365$ & $42,240,929$ & $-7.51 \%$ & $-22.96 \%$ & $9.34 \%$ & $-22.97 \%$ \\
\hline 5 & $-10 \%$ & $48,788,258$ & $46,505,426$ & $58,411,472$ & $46,505,432$ & $-11.03 \%$ & $-15.19 \%$ & $6.52 \%$ & $-15.19 \%$ \\
\hline 6 & $-5 \%$ & $49,087,739$ & $50,461,257$ & $56,924,000$ & $50,458,954$ & $-10.48 \%$ & $-7.98 \%$ & $3.81 \%$ & $-7.98 \%$ \\
\hline 7 & $0 \%$ & $54,836,062$ & $54,836,062$ & $54,836,062$ & $54,836,062$ & $0.00 \%$ & $0.00 \%$ & $0.00 \%$ & $0.00 \%$ \\
\hline 8 & $5 \%$ & $58,750,557$ & $59,602,981$ & $52,723,605$ & $59,600,670$ & $7.14 \%$ & $8.69 \%$ & $-3.85 \%$ & $8.69 \%$ \\
\hline 9 & $10 \%$ & $61,954,086$ & $63,447,698$ & $51,471,148$ & $63,447,701$ & $12.98 \%$ & $15.70 \%$ & $-6.14 \%$ & $15.70 \%$ \\
\hline 10 & $15 \%$ & $79,424,910$ & $68,339,375$ & $50,665,279$ & $68,337,051$ & $44.84 \%$ & $24.62 \%$ & $-7.61 \%$ & $24.62 \%$ \\
\hline 11 & $20 \%$ & - & $72,611,142$ & $48,666,518$ & $72,606,497$ & - & $32.41 \%$ & $-11.25 \%$ & $32.41 \%$ \\
\hline 12 & $25 \%$ & - & $76,582,948$ & $46,615,660$ & $77,418,252$ & - & $39.66 \%$ & $-14.99 \%$ & $41.18 \%$ \\
\hline 13 & $30 \%$ & - & $81,702,657$ & $45,769,928$ & - & - & $48.99 \%$ & $-16.53 \%$ & - \\
\hline
\end{tabular}

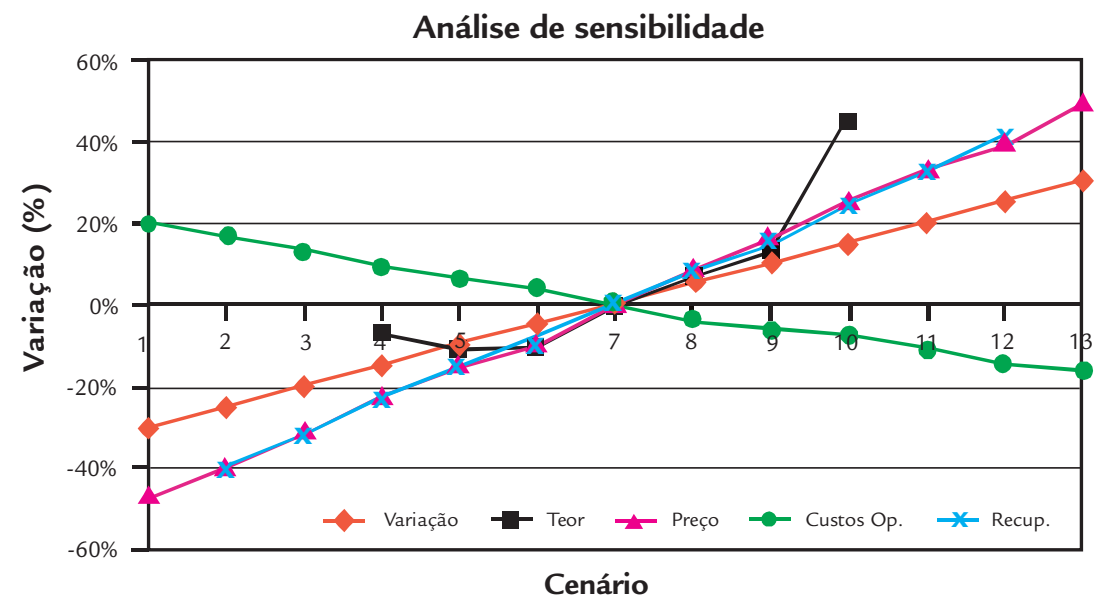

incerteza dos teores, nesse mesmo intervalo, possui um impacto da mesma ordem de grandeza de variações do preço de venda e da recuperação global. Isto significa que está demonstrado, conforme a proposta do trabalho, que as incertezas a respeito de teores não devem ser desconsideradas em análises de sensibilidade de projetos de mineração.

Dependendo da complexidade da função-benefício, as relações podem ter variações não lineares. No exemplo de aplicação da metodologia, foi usada uma função-benefício bastante simples, na qual se apresenta apenas um tipo de minério com um único tipo de processo de beneficiamento. Ainda, na lavra, foram adotados custos operacionais constantes para todos os blocos, sabendo-se da existência de variações com o incremento da profundidade da cava e, consequentemente, do aumento da distância média de transporte ou, mesmo da diferenciação de técnicas de lavra ou operações unitárias envolvidas. Ao aplicarem a otimização e o sequenciamento, observa-se que a relação sobre o VPL não segue a linearidade da penalização ou bonificação aplicada ao cenário do E-type. Esse fato significa que a aplicação direta dos percentuais, para a análise de sensibilidade, traz resultados diferentes no projeto e tal aplicação não deve ser executada apenas na fase de análise de fluxo de caixa e, sim, sobre o cálculo da função-benefício (para cima e para baixo nos parâmetros de entrada da função benefício) e os resultados dever ser, então, exportados para simulações de fluxo de caixa. 


\section{Referências bibliográficas}

COUZENS, T. R. Aspects of production planning: operating layout and phase plans, open pit mine planning and design. New York: SME-AIME, 1979. p.219-213.

DAVID, M., P. et al. Forecasting departure from planning in open pit design and grade control. APCOM, Golden, CO, 1974. p.443 - 451.

DEUTSCH, C., JOURNEL, A. G. GSLIB Geostatistical Software and User's Guide. New York: Oxford University Press, 1998. 369 p.

FORD, L. R., FULKERSON, D. R. Maximum flow through a network. Canadian Journal of Mathematics, 1956, p. 399-404.

HUTTAGOSOL, P. R., CAMERON, A computer design of ultimate pit limit by using transportation algorithm. In: 23rd APCOM, Tucson, 1992. p. 443-460.

LEMIEUX, M. Moving cone optimizing algorithm. Computer methods for the 80s in the mining industry. SME, 1979. p. 329-345

LERCHS, H., GROSSMANN, I. F. Optimum design of open pit mines. CIM Bulletin, January, 1965. p. 47-54.

PANA, M. T., CARLSON, T. R. Description of a computer technique used in mine planning of the Utab Mine of Kennnecott Copper Corp. In: 6th APCOM, 1966. p. 332-339.

PERONI, R. de L. Análise da sensibilidade do seqüenciamento de lavra em função da incerteza do modelo geológico. Porto Alegre: Programa de Pós-Graduação em Engenharia de Minas, Metalúrgica e Materiais, Universidade Federal do Rio Grande do Sul, 2002. 143 p.

TAYLOR, H. K. Mine valuation and feasibility studies. Mineral Industry Costs: Northwest Mining Association, 1977. p. 1-17.

WANG, Q., SEVIM, H. Alternative to parameterization in finding a series of maximum-metal pits for production planning. Mining Engineering, p. 178-182, 1995.

ZHAO, Y., KIM, Y. C. A new graph theory algorithm for optimal pit design. SME Transactions, p. 1832-1838, 1992.

Artigo recebido em 12 de maio de 2011. Aprovado em 22 de setembro de 2011. 